\title{
Time-Resolved Raman scattering in exfoliated and CVD graphene crystals
}

\author{
Stavros Katsiaounis ${ }^{1,2}$, Andrey V. Sharkov ${ }^{3}$, Eugeniy V. Khoroshilov ${ }^{3}$, Georgios Paterakis ${ }^{1,4}$,
} John Parthenios ${ }^{1}$, Konstantinos Papagelis ${ }^{1,5^{*}}$

${ }^{1}$ Foundation of Research and Technology Hellas, Institute of Chemical Engineering Sciences, P.O. Box 1414, GR-26504 Patras, Greece

${ }^{2}$ Department of Physics, University of Patras, GR-26504 Patras, Greece

${ }^{3}$ P.N. Lebedev Physical Institute, Russian Academy of Sciences, 119991 Moscow, Russia

${ }^{4}$ Department of Chemical Engineering, University of Patras, Patras 26504, Greece

${ }^{5}$ School of Physics, Department of Solid State Physics, Aristotle University of Thessaloniki, Thessaloniki 54124, Greece

\section{Contents}

S1. Conventional Raman spectroscopy of exfoliated and CVD graphene samples..................... 2

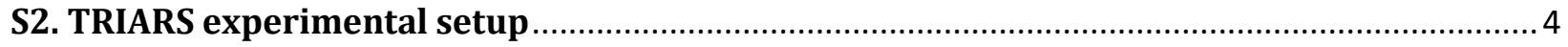

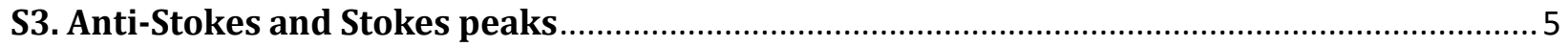

S4. TRIARS of HOPG and CVD graphene on quartz ......................................................... 7

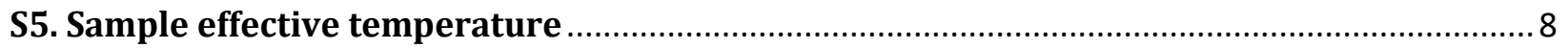

S6. G phonon lifetimes of graphite and 1-3L exfoliated graphene .......................................... 9

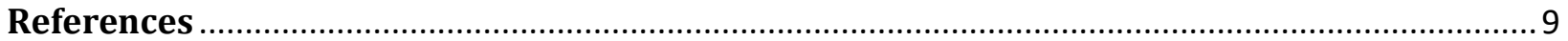




\section{S1. Conventional Raman spectroscopy of exfoliated and CVD graphene samples}

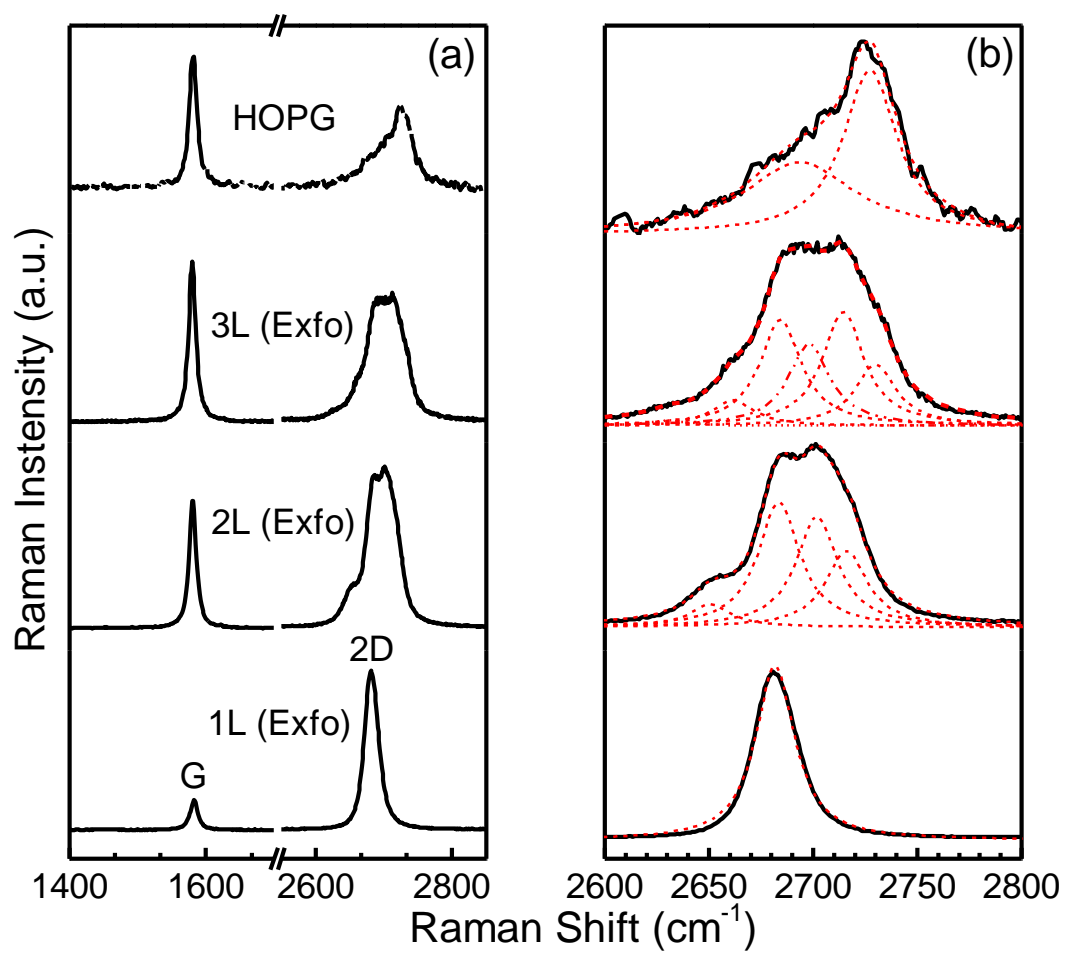

Figure S1. (a) Raman spectra of HOPG and 1-3L exfoliated graphene deposited onto $\mathrm{Si}_{2} / \mathrm{SiO}_{2}$ substrate. (b) 2D peak deconvolution with the appropriate number of Lorentzian peak functions.

The Raman spectrum of graphene is characterized by the $\mathrm{D}, \mathrm{G}$, and 2D peaks. The D peak is located at $\sim 1350 \mathrm{~cm}^{-1}$, the $\mathrm{G}$ peak at $\sim 1585 \mathrm{~cm}^{-1}$ and the $2 \mathrm{D}$ peak at $\sim 2690 \mathrm{~cm}^{-1}$. The D peak corresponds to the $\mathrm{A}_{1 \mathrm{~g}}$ breathing mode vibrations of six-membered carbon rings and activated by the conversion of neighbor $\mathrm{sp}^{2}$ hybridized carbons to $\mathrm{sp}^{3}$ ones which means the existence of defects $^{1}$. The $G$ peak comes from the stretching of the carbon-carbon bonds and it is common in all $\mathrm{sp}^{2}$ carbon materials, while the $2 \mathrm{D}$ peak is the overtone of the D-peak and it is active by a double resonance mechanism ${ }^{2}$.

Figure S1(a) illustrates typical Raman spectra of HOPG and 1-3L exfoliated graphene samples deposited on top of $\mathrm{Si} / \mathrm{SiO}_{2}$ substrates after the removal of the PMMA support film. Figure $\mathrm{S} 1$ (b) shows the appropriate number of Lorentzian peaks to fit the 2D band of the aforementioned samples $^{3-4}$. Figures S2 show typical Raman spectra of 1L and stacks of 2L and 3L CVD grown graphene deposited on $\mathrm{SiO}_{2}$ and quartz substrates after the removal of the PMMA support film.

The results of the Raman spectral parameters presented in Table S1. Regarding the monolayer graphene samples, from the $\omega_{\mathrm{G}}-\omega_{2 \mathrm{D}}$ correlation we conclude that exfoliated monolayer is in a strain and doping free state, while the transferred monolayer CVD samples on $\mathrm{Si} / \mathrm{SiO}_{2}$ and quartz experience negligible doping and biaxial strain of about $-0.05 \%$ and $0.1 \%$, respectively, due to the transferring process. 


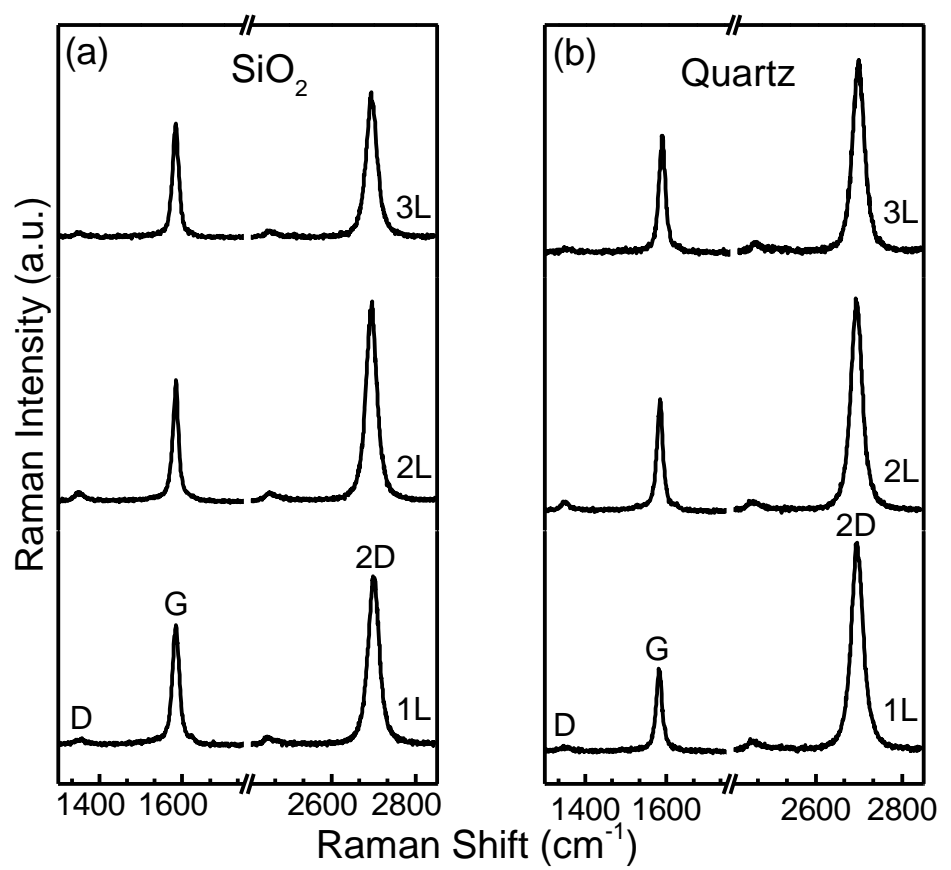

Figure S2. Raman spectra of monolayer and stacks of 2L and 3L CVD grown graphene deposited on: (a) $\mathrm{SiO}_{2}$ and (b) quartz substrates.

Table S1. Raman spectral parameters of 1-3L exfoliated graphene onto $\mathrm{Si} / \mathrm{SiO}_{2}, 1 \mathrm{~L} \mathrm{CVD}$ graphene as well as stacks of 2 and 3 layers of CVD graphene on both $\mathrm{Si} / \mathrm{SiO}_{2}$ and quartz substrates. Numbers in parentheses indicate the standard deviation derived from 150 spectra.

\begin{tabular}{ccccccc}
\hline & & $\begin{array}{c}\omega_{\mathrm{G}} \\
\left(\mathrm{cm}^{-1}\right)\end{array}$ & $\begin{array}{c}\gamma_{\mathrm{G}} \\
\left(\mathrm{cm}^{-1}\right)\end{array}$ & $\begin{array}{c}\omega_{2 \mathrm{D}} \\
\left(\mathrm{cm}^{-1}\right)\end{array}$ & $\begin{array}{c}\gamma_{2 \mathrm{D}} \\
\left(\mathrm{cm}^{-1}\right)\end{array}$ & $\mathrm{I}(\mathrm{D} / \mathrm{G})$ \\
\hline $\begin{array}{c}\text { Exfoliated } \\
\text { graphene }\end{array}$ & 1L & $1581(1)$ & $12(0.5)$ & $2680(2)$ & $22(3)$ & - \\
& 2L & $1579(1)$ & $12(1)$ & $2694(4)$ & $54(2)$ & - \\
& 3L & $1581(2)$ & $12(0.5)$ & $2702(6)$ & $57(2)$ & - \\
\hline $\mathrm{CVD} \mathrm{graphene}_{\text {on } \mathrm{SiO}_{2}}$ & $1 \mathrm{~L}$ & $1584(1)$ & $15(2)$ & $2687(2)$ & $29(3)$ & $0.26(9)$ \\
& 2L & $1583(1)$ & $17(2)$ & $2692(2)$ & $33(5)$ & $0.23(11)$ \\
& 3L & $1584(1)$ & $18(2)$ & $2691(2)$ & $36(3)$ & $0.19(9)$ \\
\hline CVD graphene & $1 \mathrm{~L}$ & $1584(2)$ & $17(4)$ & $2693(4)$ & $38(6)$ & $0.29(19)$ \\
on quartz glass & 2L & $1584(1)$ & $18(2)$ & $2694(2)$ & $33(2)$ & $0.25(13)$ \\
& 3L & $1585(1)$ & $18(1.5)$ & $2695(2)$ & $35(2)$ & $0.21(15)$ \\
\hline
\end{tabular}




\section{S2. TRIARS experimental setup}

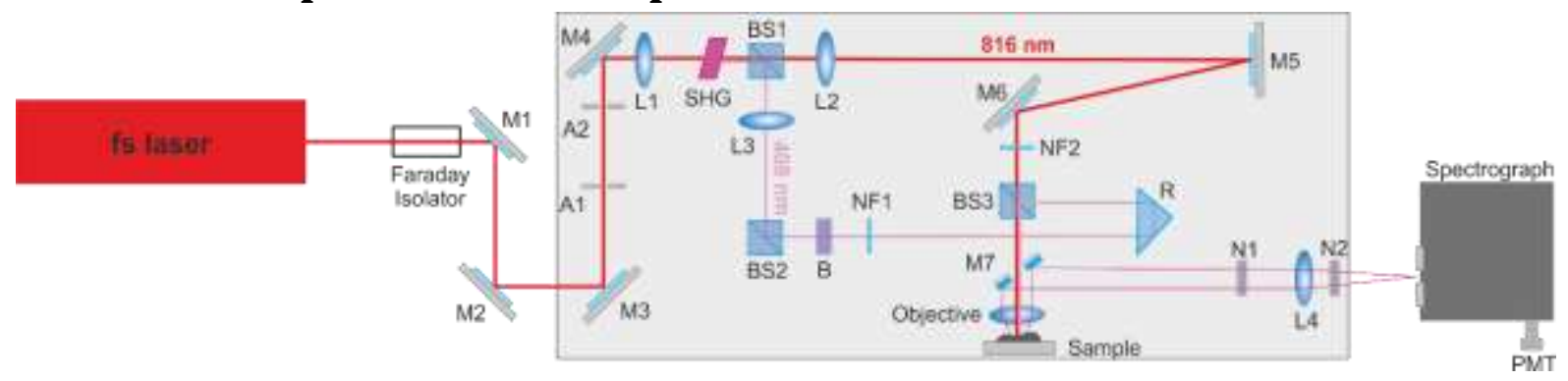

Figure S3. TRIARS experimental set-up: M1, M2, M3, M4, M5, M6 (BS1, BS2, BS3) are high reflectors at $800 \mathrm{~nm}(400 \mathrm{~nm})$. M7 is aluminum mirror with hole. L1, L2, L3, L4 are lenses and A1, A2 are apertures. SHG represents a BBO crystal for second harmonic generation. N1, N2 are notch filters. NF1, NF2 are neutral density filters. R stands for the aluminum retroreflector of the delay line and $\mathrm{B}$ is a Berek compensator.

For the generation of femtosecond pulses a Kerr Lens Mode-locking method was used. A Ti: $\mathrm{Al}_{2} \mathrm{O}_{3}$ crystal was pumped by the output of a frequency doubled diode pumped solid state (DPSS) laser providing $4 \mathrm{~W}$ in green $(532 \mathrm{~nm})$ to produce a tunable output of 720-850 nm. Femtosecond operation was initiated by jolting one of the prisms using a piston and was achieved with the combination of Kerr effect in the Ti: $\mathrm{Al}_{2} \mathrm{O}_{3}$ crystal and a soft aperture effect by the pumping beam. The laser oscillator was tuned to a bandwidth of $10 \mathrm{~nm}$ and a central wavelength of $816 \mathrm{~nm}$ generating pulses with duration of about $100 \mathrm{fs}$ and $86 \mathrm{MHz}$ repetition rate. For the compensation of the dispersive effects of the group velocity of the pulse, which limits the pulse duration, a pair of prisms was placed at Brewster angle in the cavity's long arm. A slit was used for the selection of spectral width and central wavelength. The frequency doubling of the beam was achieved with a BBO crystal through second harmonic generation effect. The pump beam $(816 \mathrm{~nm})$ and probe $(408 \mathrm{~nm})$ beams were cross polarized. The incident pump beam fluence varied from 0.44 to 1.72 $\mathrm{mJ} / \mathrm{cm}^{2}$ while the probe beam fluence kept constant at $0.18 \mathrm{~mJ} / \mathrm{cm}^{2}$. Unwanted Rayleigh scattering was excluded by the use of notch filters with a bandwidth of $\pm 2 \mathrm{~nm}$. A photomultiplier tube providing high sensitivity and ultra-fast response was used for the photon detection.

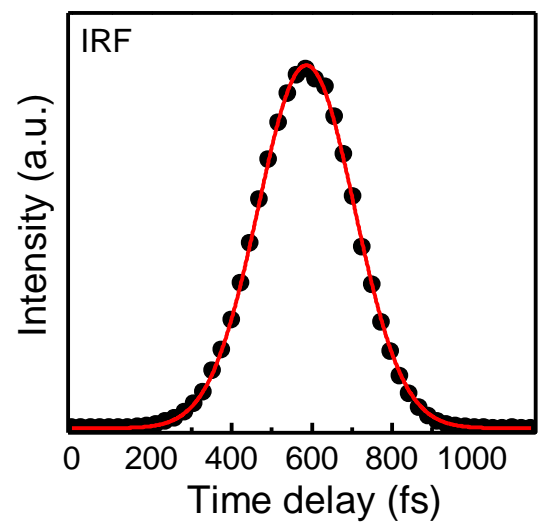

Figure S4. Time resolved Raman instrument response function (IRF) with gaussian fit. 
Figure S4 illustrates the instrument response function (IRF) of the TRIARS setup described above. As the system response is not a delta function, the determination of its IRF is necessary to correctly fit the measured transients using eq. (1) described in the main paper. To measure the IRF, an ultrafast process with duration lower than the duration of the pulse must be used. In this work, we recorded the IRF using the Third Harmonic Generation (THG) which is a non-linear parametric process that delivers a signal at one third of the excitation wavelength and can be considered as "instantaneous". THG was obtained using a second BBO crystal placed on the sample holder. The intensity of the signal was recorded as a function of the time delay. By performing a Gaussian fit to the experimental data, the FWHM ( $\tau_{w}$ ) of the IRF was found to be $280 \mathrm{fs}$.

\section{S3. Anti-Stokes and Stokes peaks}

Figure S5 illustrates the procedure followed to define the operating wavelength before recording each single wavelength kinetic decay signal. In particular, several G band anti-Stokes Raman spectra were collected (at zero delay time) and fitted with Lorentzian functions after linear background subtraction, to observe the $\mathrm{G}$ peak centre which in all cases was found to be $\sim 383.5$ $\mathrm{nm}$. It is clear from Figure S5 a relatively small change of the slope of the background as a function of fluence which could be attributed to the increase of the Rayleigh scattering with fluence.

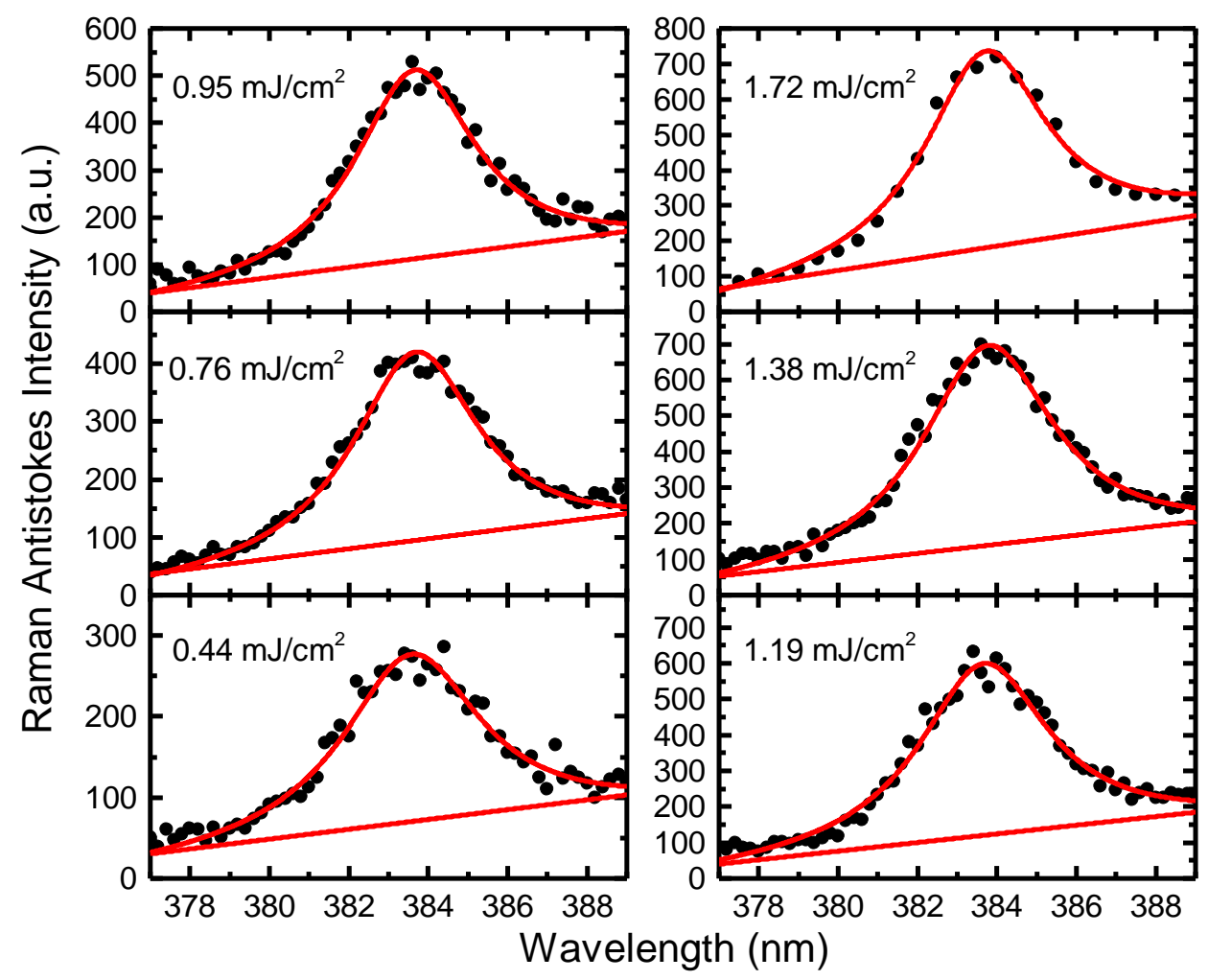

Figure S5. G anti-Stokes Raman spectra with different pump fluence values at zero delay time in graphene. 

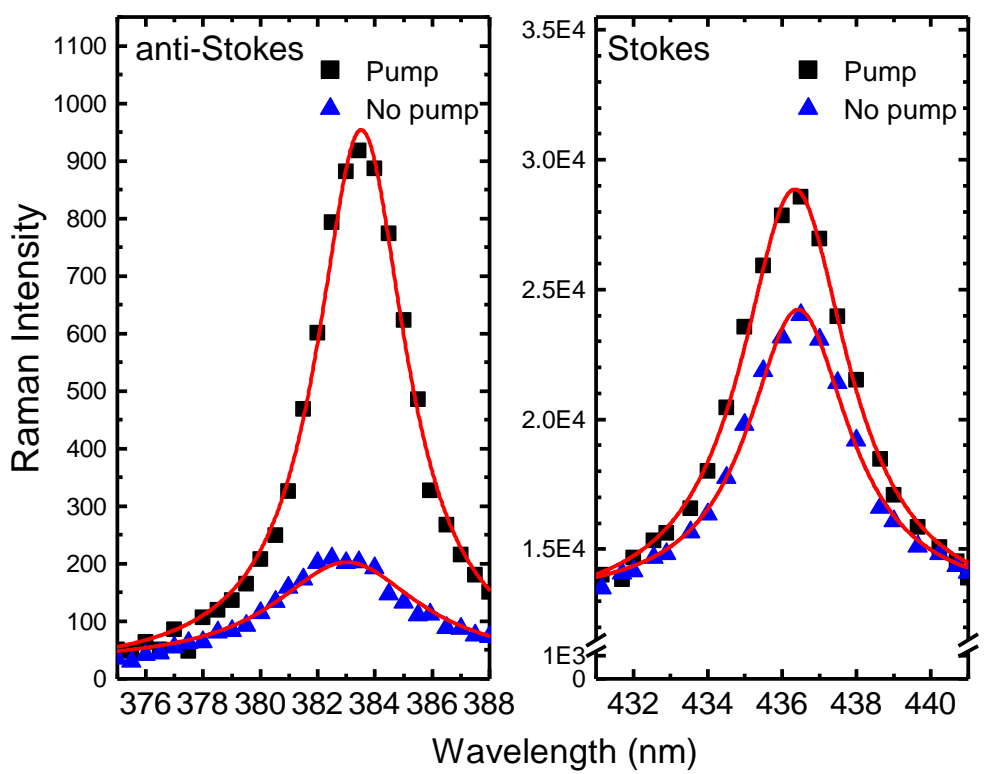

Figure S6. G anti-Stokes Raman spectra with pump and without pump excitation at zero delay time in HOPG.

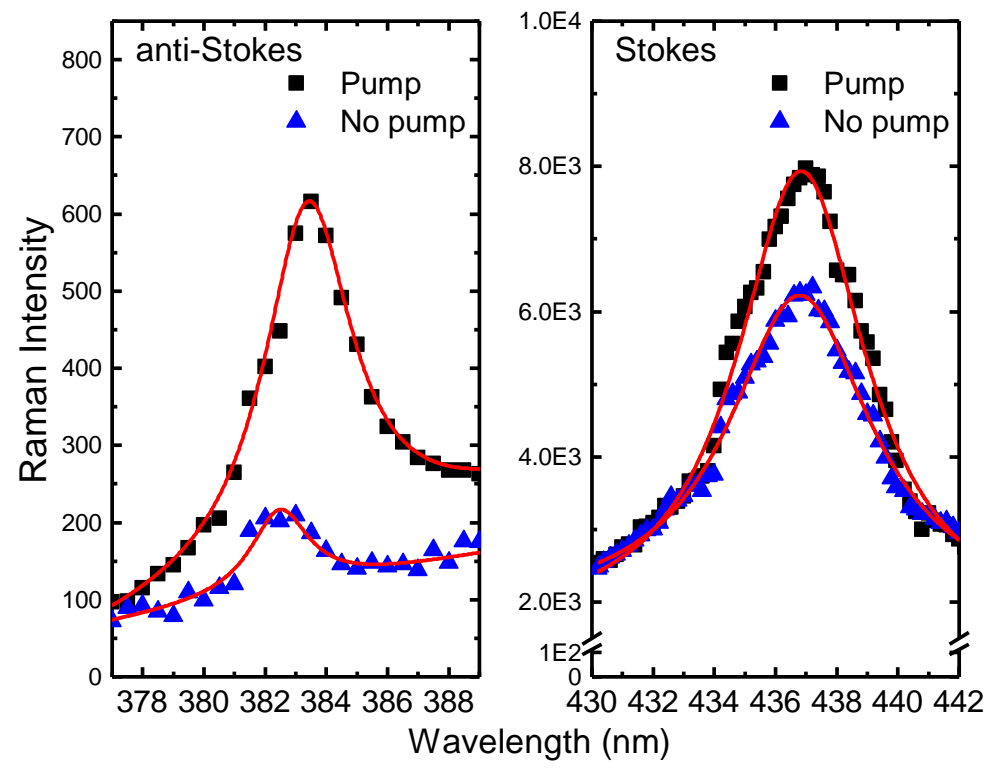

Figure S7. G anti-Stokes Raman spectra with pump and without pump excitation at zero delay time in graphene.

Figures S6 and S7 illustrate indicative anti-Stokes and Stokes spectra taken with and without pump (only probe) pulses at zero delay time. We observe a slight increase of the background to signal $(\mathrm{B} / \mathrm{S})$ ratio as the delay time increases. For example, for graphene samples the corresponding typical values are ranging between $0.20-0.26$ in the delay window $0-6 \mathrm{ps}$. Therefore, this slight variation of the $\mathrm{B} / \mathrm{S}$ ratio takes place all over the delay window and does not affect the kinetics recorded at a fixed wavelength. This is further supported by the fact that the extracted lifetime of 
graphite - its value $(2.1 \pm 0.1 \mathrm{ps})$ is broadly accepted by the graphene community- is in excellent agreement with the literature.

Also, a red shift between the peak center position of the pump anti-Stokes peak relative to the probe one is evident. The shift $v s$ delay time is not constant. The maximum shift of about $0.4 \mathrm{~nm}$ (the error in the peak position is $\sim 0.05 \mathrm{~nm}$ ) occurs at zero delay and decreases gradually at higher delay times. Also, due to the low intensity of the anti-Stokes probe peak, the error of the fitting procedure in the determination of the peak position is $\sim 0.2 \mathrm{~nm}$. By increasing the delay time the red shift varies from 0.0 (at zero delay) to $0.4 \mathrm{~nm}$ at long enough delays. On the other hand, the broad spectral profile of the G band with a FWHM of about $4.5 \mathrm{~nm}$ suggests that the intensity variation at maximum red shift (longer lifetimes) is about 3\%. Besides, as can be seen from the transients in Figures 2, S9 and S10, the noise-to-signal ratio of the recorded intensity is of the order of $1-3 \%$ at zero delay, reaching a value of $4-8 \%$ at longer delay times, depending on the sample. Therefore, the red shift only slightly affects the recorded intensities at higher delay times, while at the same time this regime exhibits considerably larger noise fluctuations. Hence, it is reasonable to conclude that the observed red shift does not affect the measurements of the lifetimes. Finally, the origin of this red shift is still unclear. One plausible explanation could be the sample thermal overheating, which is not however, supported by the magnitude of the shift ${ }^{5}$.

\section{S4. TRIARS of HOPG and CVD graphene on quartz}

Figures S8 and S9 show the time-resolved non-equilibrium dynamics of $\mathrm{G}$ phonons of a monolayer CVD graphene and a stacking of two and three CVD layers deposited on quartz glass and HOPG, respectively.

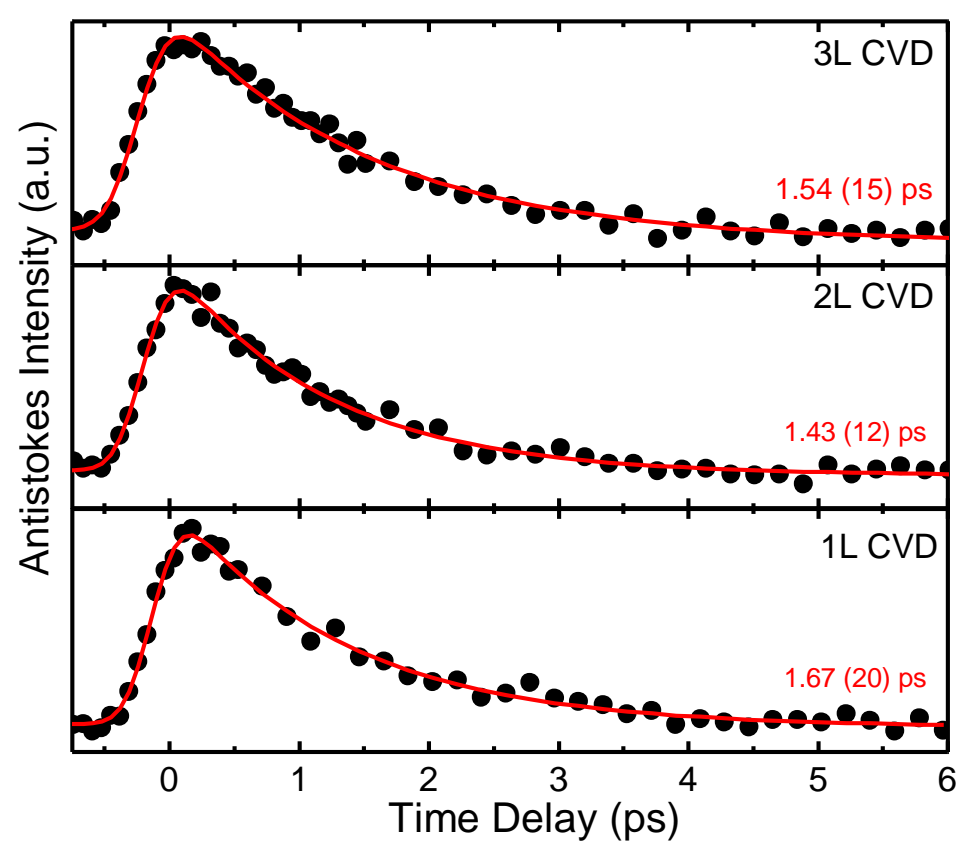

Figure S8. Room temperature normalized anti-Stokes Raman intensity for $\mathrm{G}$ phonons as a function of delay time of monolayer and decoupled 2L, 3L CVD grown graphene on quartz. Numbers in 
parentheses indicate the standard deviation extracted from ten measurements on different sample points.

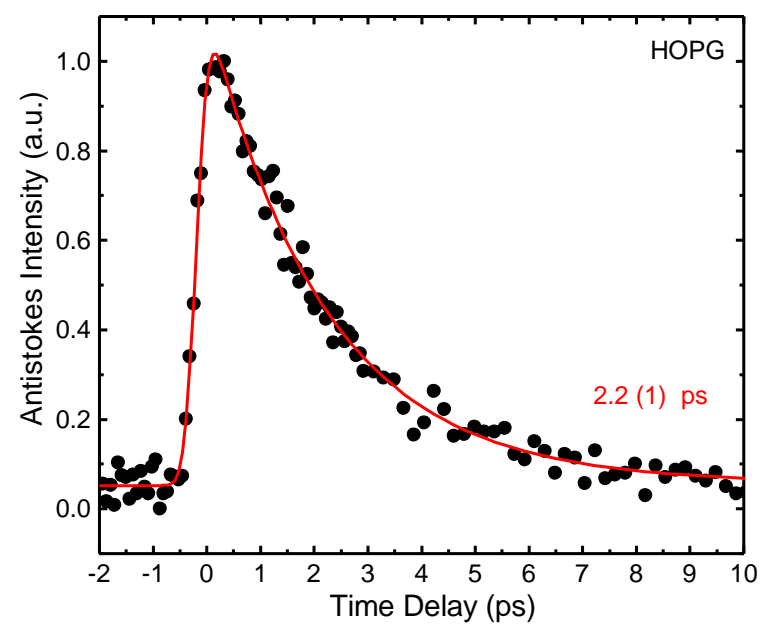

Figure S9. Room temperature normalized anti-Stokes Raman intensity for $\mathrm{G}$ phonons as a function of delay time of HOPG. Numbers in parentheses indicate the standard deviation extracted from ten measurements on different sample points.

\section{S5. Sample effective temperature}

Figure S10 illustrates the effective temperature (determined by the classical Stokes/Anti-Stokes formula written in the manuscript) as a function of pump fluence, for HOPG and graphene. A linear correlation is clear, while the extrapolated values at zero pump fluence are $470 \mathrm{~K}$ for graphene and $400 \mathrm{~K}$ for HOPG. Also, the calculated effective temperature for unpumped graphene and graphite (from Figures S6 and S7) is quite close to the above extrapolated values ( $470 \mathrm{~K}$ for graphene and $\sim 385 \mathrm{~K}$ for graphite).

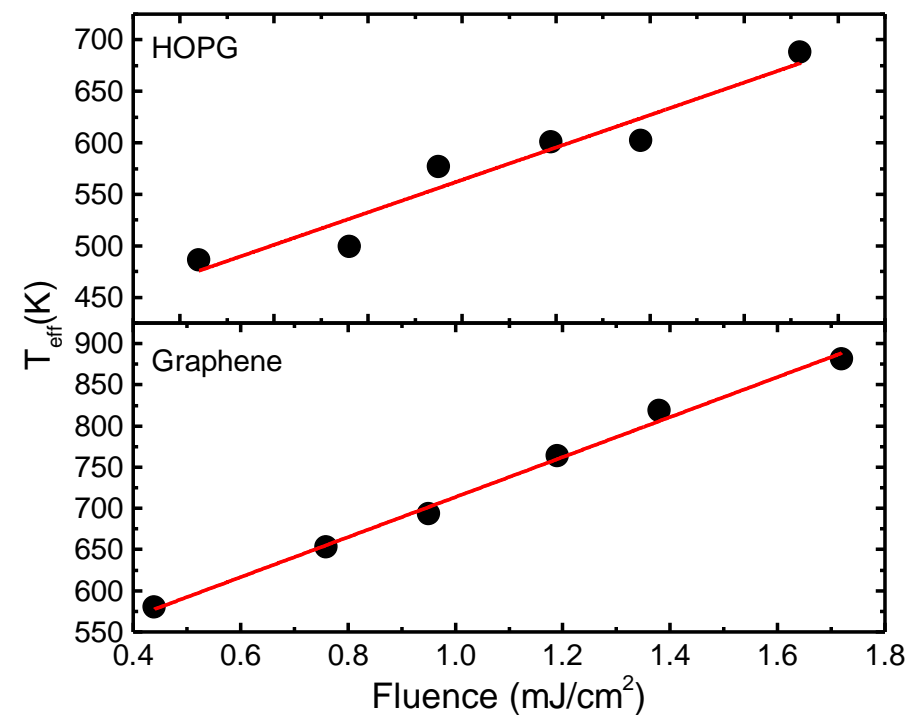

Figure S10. Effective temperature as a function of pump fluence in HOPG and graphene. 


\section{S6. G phonon lifetimes of graphite and 1-3L exfoliated graphene}

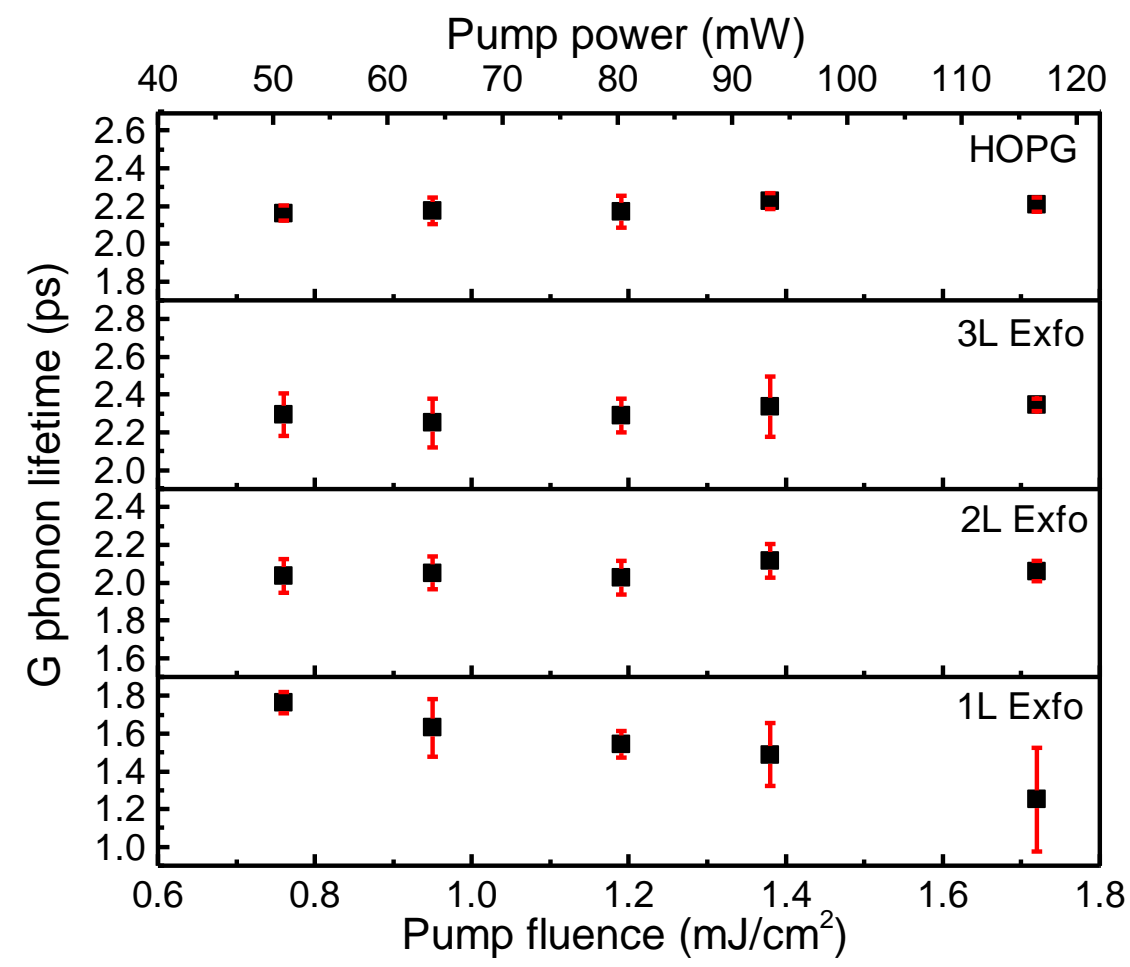

Figure S11. G-mode phonon lifetime as a function of pump fluence or average power of HOPG and 1-3L exfoliated graphene samples on $\mathrm{SiO}_{2}$ substrate.

\section{References}

1. Ferrari, A. C.; Basko, D. M., Raman spectroscopy as a versatile tool for studying the properties of graphene. Nat Nanotechnol 2013, 8, 235.

2. $\quad$ Frank, O.; Mohr, M.; Maultzsch, J.; Thomsen, C.; Riaz, I.; Jalil, R.; Novoselov, K. S.; Tsoukleri, G.; Parthenios, J.; Papagelis, K.; Kavan, L.; Galiotis, C., Raman 2D-Band Splitting in Graphene: Theory and Experiment. Acs Nano 2011, 5 (3), 2231-2239.

3. Malard, L. M.; Pimenta, M. A.; Dresselhaus, G.; Dresselhaus, M. S., Raman spectroscopy in graphene. Physics Reports 2009, 473 (5), 51-87.

4. Delikoukos, N.; Tasis, D.; Michail, A.; Parthenios, J.; Koukaras, E. N.; Papagelis, K., DopingInduced Stacking Transition in Trilayer Graphene: Implications for Layer Stacking Manipulation. ACS Applied Nano Materials 2020, 3 (12), 11861-11868.

5. Calizo, I.; Balandin, A. A.; Bao, W.; Miao, F.; Lau, C. N., Temperature Dependence of the Raman Spectra of Graphene and Graphene Multilayers. Nano Lett 2007, 7 (9), 2645-2649. 